\title{
Análise da qualidade da água da Lagoa Mirim através do IQA e de métodos estatísticos
}

\begin{abstract}
Ao longo dos anos tem-se evidenciado um aumento preocupante na degradação dos recursos hídricos em todo o mundo. Nesse cenário, criado nos EUA pela NSF, surge o IQA, como um dos métodos utilizados para o monitoramento da qualidade hídrica, utilizando variáveis que refletem as alterações ocorridas na região da bacia hidrográfica, sejam essas alterações naturais ou antrópicas. Outros métodos que podem ser utilizados para o monitoramento hídrico são os estatísticos, capazes de fornecer interpretações simples a partir da análise de dados complexos. O presente trabalho buscou, então, analisar a qualidade hídrica da Lagoa Mirim através do cálculo do IQA e de métodos estatísticos, tais como a análise de variância e análise de correlação. Para isso, foram analisados 9 parâmetros referentes a amostras coletadas em 7 pontos de monitoramento nos anos de 2015, 2016 e 2017. Os resultados obtidos mostraram a correlação entre os parâmetros de monitoramento, bem como a correlação destes parâmetros com o IQA, mostrando a relevância destes para a qualidade das águas da Lagoa Mirim. Quanto ao IQA, o recurso hídrico estudado se enquadrou, de forma geral, dentro da faixa do aceitável. Ao analisar as variações dos parâmetros e do IQA considerando como fator os meses e os anos monitorados, pode-se concluir que essas variações ocorrem de forma significativa entre esses fatores de observação. Essa conclusão pode ser corroborada pelo fato de que existem muitos fatores climáticos que podem influenciar a qualidade das águas, bem como fatores relativos a atividades agrícolas no entorno da Lagoa Mirim que também causam impactos sobre esse corpo hídrico.
\end{abstract}

Palavras-chave: Lagoa Mirim; Qualidade da água; Análise estatística; ANOVA; Correlação.

\section{Analysis of the water quality of Mirim Lagoon through WQI and statistical methods}

\begin{abstract}
Over the years there has been a worrying increase in the degradation of water resources all around the world. In this scenario, created in the USA by the NSF, WQ emerges, as one of the methods used for water quality monitoring, using variables that reflect the changes occurred in the basin region, natural or anthropic. Other methods that can be used for water monitoring are the statistical ones, capable of providing simple interpretations from the analysis of complex data. The present work then sought to analyze the water quality of Lagoa Mirim by calculating the WQI and statistical methods, such as variance and correlation. For this, 9 parameters were analyzed for samples collected at 7 monitoring points in 2015, 2016 and 2017. The results showed the correlation between the monitoring parameters, as well as the correlation of these parameters with the WQI, showing their relevance to the water quality of Mirim Lagoon. As for the IQA, the studied water resource was generally within the acceptable range. When analyzing the variations in the parameters and the IQA considering the monitored months and years as a factor, it can be concluded that these variations occur significantly between these observation factors. This conclusion can be corroborated by the fact that there are many climatic factors that can influence water quality, as well as factors related to agricultural activities in the vicinity of Lagoa Mirim that also have an impact on this water body.
\end{abstract}

Keywords: Mirim Lagoon; Water quality; Statistical analysis; ANOVA; Correlation.

Topic: Engenharia Ambiental

Reviewed anonymously in the process of blind peer.
Received: 03/01/2021

Approved: 28/01/2021
Marlon Heitor Kunst Valentini (iD Universidade Federal de Pelotas, Brasil http://lattes.cnpq.br/6499660114940771 http://orcid.org/0000-0003-3183-5142 marlon.valentini@hotmail.com

\section{Gabriel Borges dos Santos (iD}

Universidade Federal de Pelotas, Brasil http://lattes.cnpq.br/8502930511377553 http://orcid.org/0000-0002-0013-0134 gabrielwxsantos@hotmail.com

Henrique Sanchez Franz (D) Universidade Federal de Pelotas, Brasil http://lattes.cnpq.br/0985124189064768 http://orcid.org/0000-0002-7003-2687 franzhenrique@yahoo.com.br

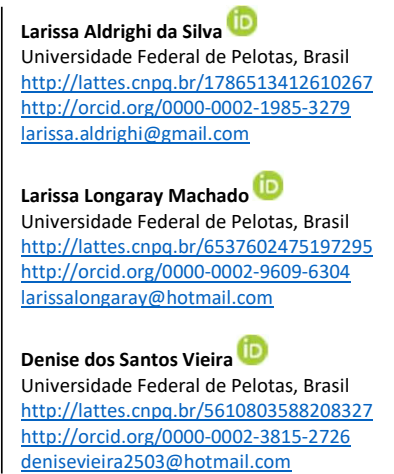

Universidade Federal de Pelotas, Brasil http://lattes.cnpq.br/1786513412610267 http://orcid.org/0000-0002-1985-3279 larissa.aldrighi@gmail.com

Larissa Longaray Machado (10)

Universidade Federal de Pelotas, Brasil http://lattes.cnpq.br/6537602475197295 ttp://orcid.org/0000-0002-9609-6304

Denise dos Santos Vieira

http://lattes conal http://orcid.org/0000-0002-3815-2726 denisevieira2503@hotmail.com

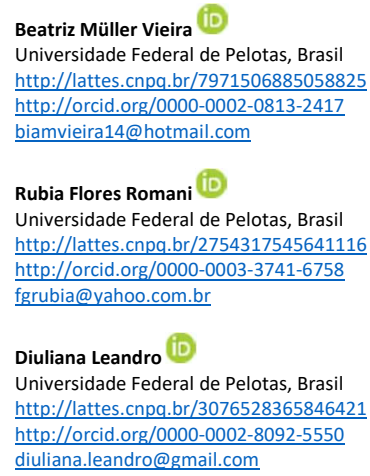

Willian Cézar Nadaleti (iD)

Universidade Federal de Pelotas, Brasil http://lattes.cnpq.br/4670559561277136 tttp://orcid.org/0000-0002-4727-4127 williancezarnadaletti@gmail.com

\section{Bruno Müller Vieira (ID)}

Universidade Federal de Pelotas, Brasil http://lattes.cnpq.br/1885554662703620 http://orcid.org/0000-0002-9615-3778 bruno.prppg@hotmail.com

DOI: 10.6008/CBPC2179-6858.2021.001.0031

\section{Referencing this:}

VALENTINI, M. H. K.; SANTOS, G. B.; MACHADO, L. L.; VIEIRA, D. S.; VIEIRA, M. B.; ROMANI, R. F.; LEANDRO, D.; NADALETII, W. C.; VIEIRA, B. M.. Análise da qualidade da água da Lagoa Mirim através do IQA e de métodos estatísticos. Revista Ibero Americana de Ciências Ambientais, v.12, n.1, p.375-384, 2021. DOI: http://doi.org/10.6008/CBPC2179-6858.2021.001.0031 


\section{INTRODUÇÃO}

Ao longo do percurso dos corpos hídricos ocorrem diversos processos que podem influenciar na qualidade hídrica. Os rios que possuem parte do seu leito em áreas urbanas, por exemplo, podem ter como uma de suas principais fontes de poluição o lançamento de efluentes industriais e esgotos domésticos (OLIVEIRA et al., 2014).

Observando de um ponto de vista histórico, tendo os centros urbanos se desenvolvido no entorno de rios e lagos de água doce, pode-se afirmar que esses recursos hídricos desempenham um grande papel no desenvolvimento das populações (OLIVEIRA, 2017). Logo, é de grande interesse que a poluição destes recursos naturais seja controlada e diminuída, corroborando, assim, a importância de que a qualidade das águas seja monitorada, bem como seus locais de poluição pontuais sejam conhecidos e tratados e que seja identificado o grupo de poluentes responsáveis por tal poluição (PIMENTA et al., 2016).

Localizada na costa atlântica da América do Sul, mais especificamente na planície costeira do Rio Grande do Sul entre o extremo sul do Brasil e o norte do Uruguai, a Lagoa Mirim é uma das lagoas de água doce mais importantes do território brasileiro, sendo o segundo maior corpo hídrico do país. Este importante recurso natural ocupa uma grande extensão territorial, sendo 375 mil hectares de superfície de água, compartidos entre os territórios brasileiros e uruguaios (OLIVEIRA et al., 2015; STEINKE et al., 2008).

Em decorrência de grande parte das terras do seu entorno serem usadas para atividades agrícolas, a Lagoa Mirim apresenta diversos focos de poluição. Estes focos, porém, também podem ser atribuídos aos lançamentos in natura de efluentes industriais e esgotos domésticos no canal São Gonçalo, um canal de aproximadamente 70 km de extensão, responsável por ligar a Lagoa Mirim à Lagoa dos Patos (SOUZA et al., 2013; OLIVEIRA et al., 2015).

Com aumento da degradação da qualidade dos recursos hídricos, surge uma maior necessidade de avaliar a qualidade das águas. Nesse cenário, desenvolvido pela National Sanitation Foundation (NSF) em 1970 nos Estados Unidos, surge o Índice de Qualidade das Águas (IQA). Esse método de avaliação da qualidade hídrica tem por objetivo comparar e monitorar as alterações na qualidade dos corpos hídricos e é definido por um conjunto de nove parâmetros considerados os mais representativos para a caracterização da qualidade hídrica (FERREIRA, 2015). No Brasil, esse índice foi adaptado pela CETESB afim de melhor se enquadrar aos recursos hídricos brasileiros e tem como objetivo principal a avaliação qualitativa de mananciais utilizados para abastecimento público (ANA, 2017).

Outra importante ferramenta que pode ser muito bem empregada para o monitoramento dos recursos hídricos é a utilização de métodos estatísticos tais como os multivariados e testes de variância. Esses métodos podem fornecer uma interpretação simples a partir de um conjunto de dados, outrora complexos, o que ajuda para um melhor entendimento dos efeitos dos processos que ocorrem no corpo hídrico estudado (HAIR et al., 2009). Um bom exemplo dos métodos citados é o uso de uma matriz de correlação, usada para correlacionar os parâmetros de qualidade analisados, bem como para suprimir os problemas de escalas desses parâmetros (BILGIN, 2015). Já no que concerne aos testes de variância, tais como o teste ANOVA, 
esses são utilizados para avaliar se os parâmetros variam de forma significativa dentro de um fator analisado, como, por exemplo, entre os anos de monitoramento (BILGIN, 2015).

Sendo assim, este estudo tem como objetivo avaliar a qualidade das águas da Lagoa Mirim através do cálculo do Índice de Qualidade das Águas e de métodos estatísticos, tais como o uso de matriz de correlação e testes de variância - ANOVA.

\section{METODOLOGIA}

\section{Região de estudo}

Este estudo foi desenvolvido abordando o corpo hídrico da Lagoa Mirim, localizado na Planície costeira do Rio Grande do Sul no extremo sul do Brasil. Esse recurso hídrico possui aproximadamente 375 mil hectares de superfície de água, constituindo-se no segundo maior corpo hídrico de características lacustres do país, ligando-se a Lagoa dos Patos por meio do canal São Gonçalo (OLIVEIRA et al., 2015).

\section{Coletas, pontos de monitoramento e análises laboratoriais}

Para fins deste estudo, com o intuito de se avaliar a qualidade da água da Lagoa Mirim, foram realizadas 22 coletas ao longo dos anos de 2015, 2016 e 2017 em 7 pontos de monitoramento, conforme descritos na Tabela 1. Estes pontos compreendem os dois lados da lagoa, sendo estes nos sentidos Pelotas Jaguarão e Pelotas - Santa Vitória do Palmar, conforme demonstrado na Figura 1.

Tabela 1: Pontos de Monitoramento da Lagoa Mirim.

\begin{tabular}{llll}
\hline Identificação & & Coordenadas Geográficas & \\
\hline LM1 & Praia Pontal & $32^{\circ} 20^{\prime} 052^{\prime \prime}$ & $052^{\circ} 49^{\prime} 21,5^{\prime \prime}$ \\
LM2 & Fazenda Bretanha & $32^{\circ} 29^{\prime} 14,0^{\prime \prime}$ & $052^{\circ} 58^{\prime} 14,9^{\prime \prime}$ \\
LM3 & Fazenda São Francisco & $32^{\circ} 38^{\prime} 25,6^{\prime \prime}$ & $053^{\circ} 08^{\prime} 56,8^{\prime \prime}$ \\
LM4 & Capilha & $32^{\circ} 29^{\prime} 23^{\prime \prime}$ & $052^{\circ} 35^{\prime} 33^{\prime \prime}$ \\
LM5 & Curral Alto & $32^{\circ} 44^{\prime} 47.41^{\prime \prime}$ & $52^{\circ} 40^{\prime} 35.99^{\prime \prime}$ \\
LM6 & Vila Anselmi & $32^{\circ} 54^{\prime} 31^{\prime \prime}$ & $052^{\circ} 48^{\prime} 08^{\prime \prime}$ \\
LM7 & Porto Santa Vitória & $33^{\circ} 29^{\prime} 51^{\prime \prime}$ & $053^{\circ} 26^{\prime} 09^{\prime \prime}$ \\
\hline
\end{tabular}

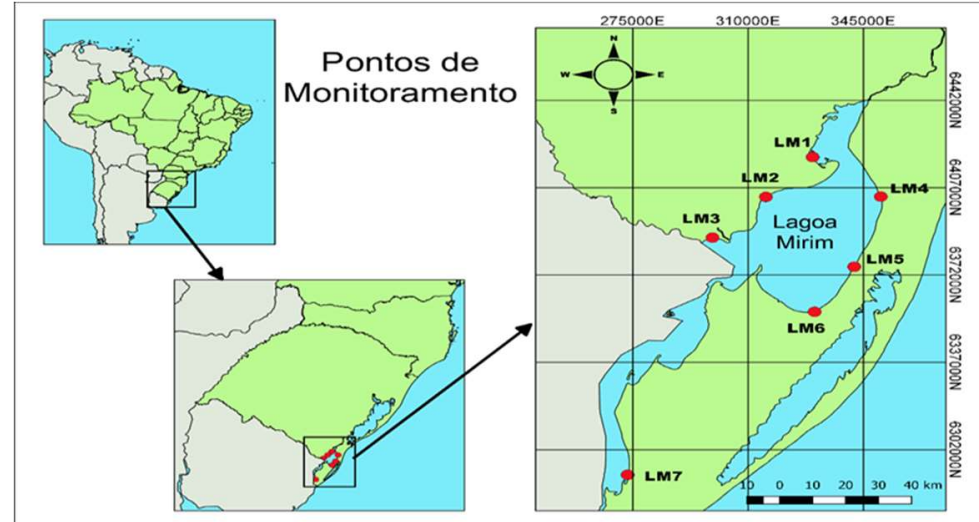

Figura 1: Pontos de Monitoramento da Lagoa Mirim.

Todas as amostras coletadas ao longo dos 7 pontos de monitoramento foram devidamente armazenas e encaminhadas para o laboratório de águas e efluentes da Agência de Desenvolvimento da Bacia Hidrográfica da Lagoa Mirim (ALM), onde foram realizadas as análises físicas, químicas e microbiológicas 
consideradas dentro do cálculo de IQA (CETESB). Todas as análises foram realizadas conforme metodologia descrita por APHA et al. (2017), sendo somente a temperatura feita in loco com o uso de termômetro digital. Os resultados dessas análises foram retirados do site da ALM, os quais estavam para acesso público durante o período abordado nesse estudo.

\section{Índice de Qualidade das Águas (IQA)}

O IQA é calculado através do produto ponderado de cada um dos seus parâmetros, conforme demonstrado na equação 1 . Este cálculo resulta em um número de 0 a 100, sendo o extremo superior considerado como de melhor qualidade.

$$
I Q A=\prod_{i=1}^{n} q i^{w i}
$$

Equação 1.

Sendo qi é a qualidade do i-ésimo parâmetro, também um número entre 0 e 100, que é obtido através do gráfico de qualidade em função da concentração medida. Já o wi é o peso do i-ésimo parâmetro representado por um número entre 0 e 1 (ANA, 2005).

Para o cálculo do IQA são realizadas as análises de 9 parâmetros conforme adaptação feita pela CETESB dos parâmetros definidos pela NSF, sendo estes: temperatura, oxigênio dissolvido, coliformes termotolerantes, $\mathrm{pH}$, demanda bioquímica de oxigênio DBO, nitrogênio total (NTK), fósforo total, turbidez e sólidos totais. Os resultados destas análises foram usados na fórmula para o cálculo do IQA para cada um dos pontos de monitoramento da Lagoa Mirim para todos os meses monitorados durante os três anos de estudo. Cada parâmetro considerado dentro do IQA possui um peso para o cálculo deste índice, conforme apresentados na Tabela 2.

Tabela 2: Pesos relativos dos parâmetros para o cálculo do IQA.

\begin{tabular}{ll}
\hline Parâmetros do IQA e seus respectivos pesos & Pesos \\
Parâmetros & 0,17 \\
Oxigênio dissolvido (OD) & 0,15 \\
Coliformes termotolerantes & 0,12 \\
Potencial hidrogeniônico (pH) & 0,10 \\
Demanda bioquímica de oxigênio & 0,10 \\
Temperatura & 0,10 \\
Nitrogênio total & 0,10 \\
Fósforo total & 0,08 \\
Turbidez & 0,08 \\
Sólidos Totais & \\
\hline
\end{tabular}

Fonte: ANA (2005).

Os resultados obtidos através do cálculo do IQA são representados em faixas de qualidade, conforme demonstrado na Tabela 3. Essas faixas variam de acordo com o estado ao qual se insere o corpo hídrico estudado, sendo adotada neste estudo a faixa relativa ao estado do Rio Grande do Sul.

Tabela 3: Classificação do IQA para o estado do Rio Grande do Sul.

\begin{tabular}{ll} 
Classificação do IQA para o RS & \\
Valor do IQA & Qualidade da água \\
$91-100$ & Ótima \\
$71-90$ & Boa \\
$51-70$ & Aceitável \\
$26-50$ & Ruim \\
$0-25$ & Péssima \\
\hline
\end{tabular}

Fonte: ANA (2005). 


\section{Análises estatísticas}

Afim de um melhor enquadramento nos testes de normalidade (K-S) e teste de KMO, no que concerne aos dados utilizados para os testes de correlação entre parâmetros, utilizou-se as médias anuais dos parâmetros de qualidade analisados em cada um dos pontos de monitoramento da Lagoa Mirim.

Com o intuito de suprimir as diferenças das ordens de grandezas dos resultados das análises laboratoriais para que não haja interferências sobre as análises estatísticas procedeu-se a padronização dos dados conforme metodologia proposta por Wilks (2006).

Afim de verificar a relação entre as variáveis estudadas, bem como dessas variáveis com o IQA da Lagoa Mirim, utilizou-se uma matriz de correlação. Segundo Reis (1997) esse tipo de análise permite identificar subconjuntos de variáveis que estão muito correlacionadas entre si.

Para saber se os dados analisados neste estudo são compatíveis com o tipo de coeficiente de correlação a ser utilizado, primeiramente foi necessário testar a normalidade destes dados. Para comprovar a normalidade dos dados utilizou-se o teste de Kolmogorov-Smirnov (K-S) com um intervalo de confiabilidade de 95\% e um nível de significância de 0,05.

Outro teste estatístico aqui utilizado, com o intuito de verificar se há variabilidade significativa dos parâmetros analisados entre os meses e/ou anos estudados, bem como a variação entre os pontos de monitoramento da Lagoa Mirim, foi o teste te variância ANOVA. Neste teste, utilizando um nível de significância de 0,05, rejeita-se a hipótese nula para aqueles que obtiverem um p-valor inferior a 0,05 e considera-se sua variabilidade como significativa dentro de uma análise estatística (BILGIN, 2015).

\section{RESULTADOS E DISCUSSÃO}

\section{Análises estatísticas}

Utilizando as médias anuais dos dados estudados foi aplicado o teste K-S para avaliar sua distribuição amostra. Segundo este teste, confirma-se a distribuição normal dos dados deste estudo, ou seja, no que diz respeito a suas médias anuais, os parâmetros utilizados obtiveram p-valor superior a 0,05 o que, segundo o teste K-S, confirma a hipótese de uma distribuição é normal. Este resultado nos permite utilizar uma matriz de correlação com coeficiente de Pearson, uma vez que para o uso deste coeficiente é necessária uma condição de normalidade amostral (GUIMARÃES, 2017).

Uma vez confirmada a normalidade da distribuição dos dados, foi criada a matriz de correlação. Essa matriz pode ser observada na Tabela 4. Salienta-se que essa matriz de correlação diz respeito as médias anuais dos dados abordados.

Considera-se uma forte correlação aquela que, em módulo, obteve coeficiente de Pearson superior a 0,5 (HELENA et al., 2000). Sendo assim, pode-se considerar, no que concerne as médias anuais dos dados analisados, como fortes as correlações conforme apresentadas na Tabela 5. 
Tabela 4: Matriz de Correlação - Médias Anuais.

\begin{tabular}{|c|c|c|c|c|c|c|c|c|c|c|}
\hline & $\mathrm{T}$ & $P$ & NTK & C. Termo & DBO & OD & Turb & $\mathrm{pH}$ & ST & IQA \\
\hline$T$ & 1 & & & & & & & & & \\
\hline $\mathrm{P}$ & 0,139 & 1 & & & & & & & & \\
\hline NTK & $-0,393$ & $-0,449$ & 1 & & & & & & & \\
\hline C. Termo & 0,106 & 0,435 & $-0,157$ & 1 & & & & & & \\
\hline DBO & $-0,38$ & $-0,071$ & 0,385 & $-0,272$ & 1 & & & & & \\
\hline OD & $-0,127$ & $-0,323$ & 0,157 & $-0,415$ & 0,593 & 1 & & & & \\
\hline Turb & 0,124 & 0,468 & $-0,116$ & 0,324 & 0,073 & 0,062 & 1 & & & \\
\hline $\mathrm{pH}$ & 0,098 & $-0,075$ & $-0,233$ & 0,342 & $-0,418$ & $-0,461$ & $-0,251$ & 1 & & \\
\hline ST & $-0,003$ & 0,697 & $-0,078$ & 0,373 & $-0,041$ & $-0,224$ & 0,623 & $-0,153$ & 1 & \\
\hline IQA & $-0,131$ & $-0,769$ & 0,376 & $-0,836$ & 0,324 & 0,478 & $-0,475$ & $-0,226$ & $-0,545$ & 1 \\
\hline
\end{tabular}

Tabela 5: Correlações Fortes.

\begin{tabular}{ll}
\hline Correlação & Coeficiente de correlação \\
\hline P - ST & 0,697 \\
P - IQA & $-0,769$ \\
C. Termo - IQA & $-0,836$ \\
DBO - OD & 0,593 \\
ST - Turb & 0,623 \\
ST - IQA & $-0,545$ \\
\hline
\end{tabular}

Analisando as correlações consideradas como fortes, destacam-se aquelas que fazem correlação com o IQA. Pode-se observar que as variáveis P, C. Termo e ST possuem uma forte correlação negativa com o índice de qualidade da água da Lagoa Mirim, significando que quanto maior estas variáveis, menor o IQA. Isso corrobora-se pelo fato de que estas variáveis podem ser advindas de fontes de poluição agrícola e/ou de esgotos domésticos (ANA, 2005).

Já no que diz respeito as correlações entre P e ST, DBO e OD, bem como ST e Turb, essas apresentamse como fortes correlações positivas, ou seja, de regra geral, suas concentrações aumentam ou diminuem na mesma direção. No que diz respeito a correlação DBO - OD, essa pode ser explicada pelo fato de que o oxigênio dissolvido pode ser influenciado pelo lançamento de efluentes na água (PINTO, 2007) e a DBO fornece um indicativo da carga orgânica do recurso hídrico (SPERLING, 2005). Já a correlação P - ST pode ser atribuída ao fato de que ambos podem ser oriundos de atividades agrícolas (ROCHA et al., 2016). No que concerne a correlação ST - Turb, está ocorre pelo fato de que os ST contribuem para a turbidez da água (MACEDO et al., 2018). Para melhor entender essas correlações, também foram analisadas suas significâncias. Para um p-valor inferior a 0,05, consideram-se significantes as correlações. Esses resultados são apresentados na Tabela 6.

Tabela 6: Significância das correlações.

\begin{tabular}{|c|c|c|c|c|c|c|c|c|c|c|}
\hline & $T$ & $P$ & NTK & C. Termo & DBO & OD & Turb & $\mathrm{pH}$ & ST & IQA \\
\hline \multicolumn{11}{|l|}{$T$} \\
\hline$P$ & 275, & & & & & & & & & \\
\hline NTK & ,039 & ,021 & & & & & & & & \\
\hline C. Termo & ,324 & ,024 & 249, & & & & & & & \\
\hline DBO & 044, & 380, & 042, & 117, & & & & & & \\
\hline OD & 291 & ,077 & 249 & ,031 & ,002 & & & & & \\
\hline Turb & 296 & ,016 & 309 & ,076 & 377 & 394 & & & & \\
\hline $\mathrm{pH}$ & ,336 & ,374 & 154 & ,065 & ,030 & ,018 & 136 & & & \\
\hline ST & ,495 & ,000 & 369 & ,048 & ,429 & 165 & ,001 & ,254 & & \\
\hline IQA & ,285 & ,000 & ,046 & ,000 & ,076 & ,014 & ,015 & , 162 & ,005 & \\
\hline
\end{tabular}

Quanto as correlações consideradas fortes, todas elas foram significantes. Porém, algumas correlações com coeficiente de Pearson inferior a 0,5 também obtiveram um p-valor inferior a 0,05 atestando 
sua significância. Isso mostra que certos parâmetros, apesar de não demonstrarem uma correlação dita como forte, ainda influenciam significativamente sobre outros.

Exemplifica-se isso utilizando a correlação entre P e NTK. Ao considerarmos apenas o coeficiente de correlação de Pearson, essas duas variáveis não possuem forte correlação entre si. Porém, ao analisar a significância dessa correlação, observa-se que essas variáveis influenciam significativamente uma com a outra, porém em menor intensidade.

\section{Índice de Qualidade da Água}

Os resultados de IQA das médias anuais de todos os pontos de monitoramento da Lagoa Mirim, são apresentados na Tabela 7.

Tabela 7: Resultados anuais de IQA.

\begin{tabular}{llll}
\hline Pontos de Monitoramento & \multicolumn{2}{l}{ Médias Anuais } & \\
\cline { 2 - 4 } & 2015 & 2016 & 2017 \\
\hline LM1 & 68,7 & 69,9 & 62,1 \\
LM2 & 64,7 & 68,8 & 60,3 \\
LM3 & 67,0 & 71,9 & 60,1 \\
LM4 & 71,7 & 67,7 & 58,8 \\
LM5 & 58,3 & 62,7 & 60,2 \\
LM6 & 76,0 & 68,4 & 56,5 \\
LM7 & 68,7 & 74,9 & 70,0 \\
\hline
\end{tabular}

Como pode ser observado na Tabela 7, os maiores resultados de IQA ocorreram no ano de 2016, à exceção dos pontos LM4 e LM6 que tiveram maiores resultados no ano de 2015. Já os piores resultados se encontram em sua maioria no ano de 2017 exceto nos pontos LM5 e LM7, onde os piores resultados ocorrem no ano de 2015.

Ao comparar os pontos de monitoramento entre si, os maiores resultados ocorreram no ponto LM7 exceto no ano de 2015, que demonstrou um melhor IQA no ponto LM6. Já os piores resultados se encontram no ponto LM5, menos pelo ano de 2017 que teve como menor IQA o ponto LM6. Essas variações podem ser melhor observadas na figura 2.

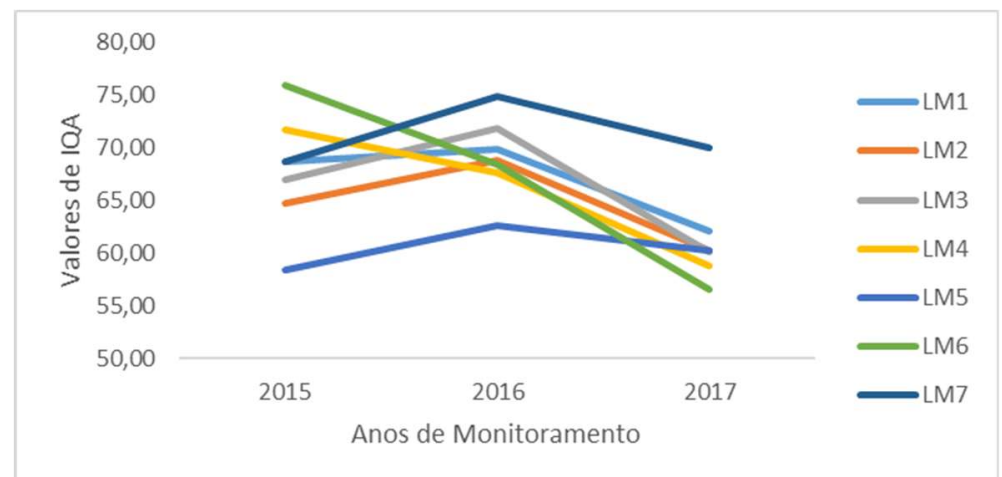

Figura 2: Gráfico das Médias Anuais de IQA.

Nenhum dos pontos de monitoramento, no período de tempo estudado, obteve um resultado de IQA abaixo da faixa do aceitável (que varia de 51 a 70), bem como nenhum destes obteve uma classificação ótima (91 a 100), conforme a classificação da Tabela 3 (ANA, 2005). O fato de não serem encontradas médias 
anuais dentro da faixa ótima pode ser explicado ao analisar as áreas do entorno desse recurso hídrico, bem como das atividades realizadas nessas áreas, sendo predominantemente agrícolas.

\section{Análise de Variância (ANOVA) - Parâmetros e IQA}

Para melhor entender o comportamento do recurso hídrico aqui estudado, além das análises e resultados já discutidos, foram realizadas análises de variâncias sobre cada um dos parâmetros estudados e do próprio IQA em relação aos meses e anos de estudo, bem como sua variância entre os pontos de monitoramento. Para essas análises de variância foram considerados juntos todos os pontos de monitoramento ao longo de todos os meses e anos estudados, dentro de uma mesma análise estatística. Esses resultados são apresentados na Tabela 8.

Tabela 8: Análise de Variância (ANOVA) dos resultados dos parâmetros e do IQA.

\begin{tabular}{llll}
\hline ANOVA & \multicolumn{3}{l}{} \\
\hline & Sig. (p-valor) & \\
\cline { 2 - 4 } Entre Ponto & Anual & Mensal \\
\cline { 2 - 4 } T & 0,000 & 0,005 & 0,000 \\
P & 0,355 & 0,001 & 0,005 \\
NTK & 0,400 & 0,107 & 0,058 \\
C. Termo & 0,492 & 0,002 & 0,000 \\
DBO & 0,797 & 0,000 & 0,000 \\
OD & 0,064 & 0,000 & 0,000 \\
Turb & 0,046 & 0,981 & 0,001 \\
pH & 0,390 & 0,001 & 0,000 \\
ST & 0,002 & 0,000 & 0,478 \\
IQA & 0,043 & 0,000 & 0,000 \\
\hline
\end{tabular}

No que diz respeito a variância dos parâmetros e dos IQA's entre os pontos de monitoramento da LM, como é possível observar na Tabela 8, somente os parâmetros T, Turb, ST e o próprio IQA possuem variabilidade significativa. Já no que concerne as variabilidades anuais e mensais os resultados apresentam um comportamento diferente.

Analisando a variabilidade anual, todos os parâmetros, exceto NTK e Turbidez, variam significativamente. Já avaliando a variabilidade mensal, retêm-se a hipótese nula, que considera a variabilidade como não significativa, para os parâmetros NTK e ST. A hipótese nula para este teste é definida como não havendo variabilidade significativa entre os fatores analisados e, para um nível de significância de 0,05, conforme já dito anteriormente, ela é rejeitada para os p-valores inferiores a 0,05.

Quando avaliada a variabilidade entre os pontos de monitoramento como não significativa, em sua maioria, podemos atribuir isto ao fato de serem todos pontos de monitoramento de um mesmo recurso hídrico que sofre as mesmas pressões naturais e antrópicas. Ou seja, os mesmos fatores que contribuem para a qualidade das águas deste recurso hídrico se fazem presente por toda a sua extensão.

Considerando que há muitos fatores climáticos que podem influenciar a qualidade das águas bem como fatores advindos da agricultura, tais como épocas de colheita e as diferentes safras cultivadas, vê-se corroborado o fato de que há variabilidade significativa na grande maioria dos parâmetros ao analisar a variação mensal e/ou anual para o corpo hídrico como um todo.

Estes resultados servem para certificar a importância de monitorar a qualidade hídrica, bem como 
os parâmetros responsáveis por este índice, mensal e anualmente. Levando em consideração o fato de haver variabilidade significativa entre os pontos de monitoramento, no que diz respeito aos seus IQA's, pode-se, também, inferir a importância da análise destes pontos, pois diagnosticando os pontos de pior qualidade pode-se melhor direcionar os esforços de controle à poluição.

\section{CONCLUSÕES}

Através dos resultados obtidos nesse estudo conclui-se que foi possível avaliar a qualidade das águas da Lagoa Mirim através do cálculo do IQA e do uso de métodos estatísticos, tais como analises de variância e de correlação.

No que concerne à variação da qualidade entre os pontos de monitoramento, medida através do IQA, pode-se concluir, conforme demonstrado pelo teste ANOVA, que essa variação ocorre de forma significativa. Ao analisar as variações dos parâmetros e do IQA considerando como fator os meses e os anos monitorados, pode-se concluir que, também, essas variações ocorrem de forma significativa entre esses fatores de observação. Essa conclusão pode ser corroborada pelo fato de que existem muitos fatores climáticos, tais como temperatura e pluviometria, que podem influenciar a qualidade das águas, bem como fatores relativos a atividades agrícolas no entorno da Lagoa Mirim que também causam impactos sobre esse corpo hídrico.

Para melhor entender os processos que interferem na qualidade do recurso hídrico aqui estudado, sugere-se a análise de uso e ocupação do solo nas áreas do entorno da Lagoa Mirim. Esse tipo de análise permite inferir quais atividades ocorrem nas áreas adjacentes a lagoa, corroborando, assim, para um entendimento mais amplo dos fatores de poluição que ali ocorrem.

\section{REFERÊNCIAS}

ANA. Agência Nacional das Águas. Indicadores de qualidade: índice de qualidade das águas. Brasília: ANA, 2017.

ANA. Agência Nacional das Águas. Caderno de Recursos Hídricos: 1 Panorama da qualidade das águas superficiais no Brasil. Brasília: TDA Desenho e Arte Ltda., 2005.

APHA; AWWA; WEF. American Public Health Association American Water Works Association; Water Environment Federation. Standard methods for the examination of water and wastewater. 23 ed. Washington: APHA, 2017.

BILGIN, A.. An assessment of water quality in the Coruh Basin (Turkey) using multivariate statistical techniques. Environmental Monitoring and Assessment, v.187, n.721, 2015.

FERREIRA, K. C. D.; LOPES, F. B.; ANDRADE, E. M.; MIIRELES, A. C. M.; SILVA, G. S.. Adaptação do índice de qualidade da água da National Sanitation foundation ao semiárido brasileiro. Revista Ciência Agronômica, v.46, n.2, p.277-286, 2015.

GUIMARÃES, P. R. B.. Análise de Correlação e medidas de associação. Curitiba: Universidade Federal do Paraná, 2017.
HAIR, J. F.; BLACK, W. C.; BABIN, B. J.; ANDERSON, R. E.; TATHAM, R. L.. Análise multivariada de dados. São Paulo: Bookman, 2009.

HELENA, B.; PARDO, R.; VEGA, M.; BARRADO, E.; FERNÁNDEZ, J. M.; FERNÁNDEZ, L.. Temporal evolution of groundwater composition in na alluvial aquifer (Pisuerga River, Spain) by principal component analysis. Water Research, v.34, p.807816, 2000.

MACEDO, T. L.; REMPEL, C.; MACIEL, M. J.. Análise FísicoQuímica e Microbiológica de água de poços artesianos em um município do Vale do Taquari-RS. Tecno-Lógica, Santa Cruz do Sul, v.22, n.1, p.58-65, 2018.

OLIVEIRA, L. N.; SILVA, C. E.. Qualidade da água do rio Poti e suas implicações para atividade de lazer em Teresina/PI. Revista Equador, v.3, n.1, p.128-147, 2014.

OLIVEIRA, M. L. V. M.. Gestão de águas, territórios e desenvolvimento econômico. ACTA Geográfica, Boa Vista, v.11, n.27, p.42-61, 2017. 
OLIVEIRA, H. A.; FERNANDES, E. H. L.; MÖLLER JUNIOR, O. O.; COLLARES, G. L.. Processos hidrológicos e hidrodinâmicos da Lagoa Mirim. Revista Brasileira de Recursos Hídricos, v.20, n.1, p.34-45, 2015

PIMENTA, R. H. O.; REIS, S. P.; FONSECA, M.. Diagnóstico ambiental em três trechos distintos do córrego Capão Regional Venda Nova, município de Belo Horizonte, MG. Revista PETRA, v.2, n.1, p.153-173, 2016.

PINTO, M. C. F.. Manual medição in loco. Temperatura, pH, Condutividade Elétrica e Oxigênio Dissolvido. 2007.

\section{REIS, E.. Estatística multivariada aplicada. Lisboa, 1997.}

ROCHA, C. H. B.; PEREIRA, A. M.. Análise multivariada para seleção de parâmetros de monitoramento em manancial de Juiz de Fora, Minas Gerais. Revista Ambiente \& Água, v.11, n.1, p.176-187, 2016.
SOUZA, M. F.; MEDRONHA, G. A.; MILANI, I. C.

Monitoramento da qualidade da água de um manancial hídrico sob influência de atividades antrópicas, no município de pelotas, RS-Brasil. In: SIMPÓSIO BRASILEIRO DE RECURSOS HÍDRICOS. Anais. 2013.

SPERLING, M. V.. Introdução à Qualidade das Águas e ao Tratamento de Esgotos. 3 ed. Belo Horizonte: Departamento de Engenharia Sanitária e Ambiental, 2005.

STEINKE, V. A.; SAITO, C. H.. Exportação de carga poluidora para identificação de áreas úmidas sob risco ambiental na bacia hidrográfica da Lagoa Mirim. Sociedade \& Natureza, v.20, n.2, p.43-67, 2008.

WILKS, D. S.. Statistical Methods in the atmospheric sciences. 2 ed. International geophysics series 91, 2006. 